\title{
Generation of hyperentangled states and two-dimensional quantum walks using $J$ or $q$ plates and polarization beam splitters
}

\author{
P. A. Ameen Yasir $\circledast^{1, *}$ and C. M. Chandrashekar ${ }^{1,2,3, \dagger}$ \\ ${ }^{1}$ Department of Instrumentation and Applied Physics, Indian Institute of Science, Bengaluru 560012, India \\ ${ }^{2}$ The Institute of Mathematical Sciences, C. I. T. Campus, Taramani, Chennai 600113, India \\ ${ }^{3}$ Homi Bhabha National Institute, Training School Complex, Anushakti Nagar, Mumbai 400094, India
}

(Received 29 September 2021; accepted 3 January 2022; published 18 January 2022)

\begin{abstract}
A single photon can be made to entangle simultaneously in its different internal degrees of freedom (DoF)polarization, orbital angular momentum (OAM), and frequency-as well as in its external DoF-path. Such entanglement in multiple DoF is known as hyperentanglement and provides additional advantage for quantum information processing. We propose a passive optical setup using $q$ plates and polarization beam splitters to hyperentangle an incoming single photon in polarization, OAM, and path DoF. By mapping polarization DoF to a two-dimensional coin state, and path and OAM DoF to two spatial dimensions, $x$ and $y$, we present a scheme for realization of a two-dimensional discrete-time quantum walk using only polarization beam splitters and $q$ plates ensuing the generation of hyperentangled states. The amount of hyperentanglement generated is quantified by measuring the entanglement negativity between any two DoF. We further show that hyperentanglement generation can be controlled by using an additional coin operation or by replacing the $q$ plate with a $J$ plate.
\end{abstract}

DOI: 10.1103/PhysRevA.105.012417

\section{INTRODUCTION}

It is possible to entangle photons in more than one degree of freedom (DoF) such as polarization, time energy, path, orbital angular momentum (OAM), and so on [1-3]. Such states are called hyperentangled states [1]. Due to extension in the dimension of the Hilbert space of such paired photons, increase in the channel capacity has been demonstrated [4] and as a consequence hyperentanglement is poised to offer additional quantum advantage. Hyperentanglement in polarization and path DoF has been exploited in the context of entanglement purification protocols, which has found applications in entanglement-based quantum key distribution [5-8]. Single photons can also be simultaneously entangled in polarization, path, and OAM DoF. While polarization and OAM correspond to internal DoF [9] of the photon, path DoF corresponds to external DoF. The amount of entanglement between these three DoF can be, for instance, generated and controlled using devices such as waveplates (both quarter and half-waveplates), polarization beam splitters (PBS), and $q$ plates [10] or $J$-plates [11]. A single photon entangled in these three DoF can also be thought of as a quantum walker in higher dimensions. For example, in a discrete-time quantum walk in two spatial dimensions, a coin degree of freedom can be mapped to polarization DoF and the two spatial dimensions can be mapped to path and OAM DoF. Therefore, controlled engineering of interactions between different DoF of a single photon to generate and control hyperentanglement

\footnotetext{
*ameenyasir@iisc.ac.in

†chandracm@iisc.ac.in
}

can be directly mapped to the controlled realization of higherdimensional quantum walks.

Quantum walks, the quantum analog of classical random walks, are broadly classified into two categories : discretetime quantum walk (DTQW) and continuous-time quantum walk (CTQW) [12]. In the former case, the quantum coin dictates the direction in which the walker moves and the walk evolves in the Hilbert space $\mathcal{H}_{c} \otimes \mathcal{H}_{p}$, where $\mathcal{H}_{c}$ denotes the Hilbert space corresponding to the coin space and $\mathcal{H}_{p}$ denotes the position space in which the walker moves. In the case of CTQW, no coin operation is necessary, and the state evolves only in the position Hilbert space.

For a one-dimensional DTQW the Hilbert space $\mathcal{H}_{c}$ is spanned by two-dimensional (2D) basis vectors $|H\rangle=[1,0]^{T}$ and $|V\rangle=[0,1]^{T}$. It can be noted that $|H\rangle$ and $|V\rangle$ denote Jones vectors corresponding to horizontal and vertical polarization states of photons, respectively. The Hilbert space $\mathcal{H}_{p}$ is spanned by the position basis $\{|x\rangle\}$, where $x \in \mathbb{Z}$. Each step of DTQW can be described using a composition of quantum coin operation $\hat{C}_{\sigma}$ in $\mathrm{SU}(2)$,

$$
\hat{C}_{\sigma}=\left[\begin{array}{cc}
e^{i \xi} \cos \theta & e^{i \zeta} \sin \theta \\
-e^{-i \zeta} \sin \theta & e^{-i \xi} \cos \theta
\end{array}\right]
$$

on $\mathcal{H}_{c}$, followed by a position shift operation,

$$
\hat{S}=\sum_{x=-\infty}^{\infty}[|H\rangle\langle H|\otimes| x-1\rangle\langle x|+| V\rangle\langle V|\otimes| x+1\rangle\langle x|]
$$

on the combined Hilbert space [13]. After each step of walk operation the walker will evolve in superposition of position space entangling the two Hilbert spaces. In an optical setting with polarization DoF, any $\hat{C}_{\sigma}$ in $\mathrm{SU}(2)$ can be realized using two quarter waveplates and a half waveplate [14] and $\hat{S}_{x}$ can 
be realized using PBS. In general, the one-dimensional (1D) DTQW evolution after $n$ steps can be given by

$$
\begin{aligned}
\left|\Psi_{n}\right\rangle & =\left[\hat{S}_{x}\left(\hat{C}_{\sigma} \otimes \mathbb{1}_{x}\right)\right]^{n}\left|\Psi_{\text {in }}\right\rangle \\
& =\sum_{x=-\infty}^{\infty}\left[a_{x}^{(n)}|H\rangle+b_{x}^{(n)}|V\rangle\right] \otimes|x\rangle,
\end{aligned}
$$

where $\left|\Psi_{\text {in }}\right\rangle$ is the initial state, $\mathbb{1}_{x}$ refers to identity operator in the position space, and $a_{x}^{(n)}$ and $b_{x}^{(n)}$ are normalized complex coefficients. The evolved state is evidently entangled in coin and spatial DoF.

For extension of DTQW to the 2D space, the Hilbert space will be a composition of $\mathcal{H}_{c} \otimes \mathcal{H}_{p_{x}} \otimes \mathcal{H}_{p_{v}}$, where $\mathcal{H}_{c}$ corresponds to the coin space and $\mathcal{H}_{p_{x}}$ and $\mathcal{H}_{p_{y}}$ refer to the Hilbert spaces corresponding to the position spaces in the $x$ and $y$ directions, respectively. Since the state has to simultaneously evolve in both $x$ and $y$ spaces, it is natural to expect the use of four-dimensional coin space and a corresponding coin operation. Two well-known examples of such coin choices are Grover coin and four-dimensional discrete-Fourier transform coin [15]. However, it was shown that such 2D DTQW can as well be implemented using just 2D coin operation [16-18]. For instance, the Grover walk with an initial state $\left(\frac{1}{2}\right)(|0\rangle-$ $|1\rangle-|2\rangle+|3\rangle) \otimes|x=0\rangle \otimes|y=0\rangle$ can be implemented using a two-state alternate walk, in which a two-dimensional coin operation is used and each step of the walk is split into an evolution in one dimension followed by an evolution in the other dimension. It has also been demonstrated that the alternate walk can be implemented in the form of a Pauli walk, where Pauli operators' bases are used as conditions in the shift operators and no coin operation is therefore necessary [18].

There has been a continued interest in efficient implementation of quantum walks (both in 1D and 2D spaces) in various quantum systems. For example, in $1 \mathrm{D}$, quantum walk has been realized using physical systems such as NMR [19], optical lattice [20], linear optical devices [21-24], ion traps [25,26], and $q$ plates (single photons [27] as well as bright classical light $[28,29])$, to name a few. In 2D, the quantum walk has been realized using photonic waveguide arrays [30], liquid-crystal devices [31], etc. Various new schemes have been proposed for the realization of 1D quantum walk which include $q$ plates and waveplates [32], passive optical devices [33,34], and cross-Kerr nonlinearity [35].

Inspired by the Pauli walk where different bases are used for evolution in different spatial dimensions, in this paper, we propose a passive optical setup-using $J$ plates [11] or $q$ plates [10] and PBS) - to generate hyperentanglement in polarization, path, and OAM DoF of a single photon. Here, $J$ plates or $q$ plates will be used to control the OAM [36] and polarization DoF, while PBS will be used to control the path DoF. Upon evolution, we show that the photon will be hyperentangled in these three DoF. This setup can also effectively simulate a 2D modified form of Pauli walk in OAM and position DoF where coin operation is not required. Due to a basis change that the $J(q)$ plate and PBS introduce, the effect of coin operation in the path dimension is absorbed into the $J(q)$ plate and the effect of coin operation in OAM dimension is absorbed into PBS. By mapping the path and
OAM DoF to $x$ and $y$ dimensions we can recover the DTQW in two-dimensional position space.

This paper is organized as follows. In Sec. II we briefly review schemes for realizing 2D DTQW such as Pauli and alternate walks and explain how the evolved state is hyperentangled in the associated Hilbert spaces. In Sec. III we propose a passive optical setup to hyperentangle the incoming single photon in the three DoF (polarization, path, and OAM). This hyperentanglement is quantified by measuring the entanglement negativity between any two of the three DoF. We then present our numerical results by simulating the two-dimensional modified Pauli walk, which does not require an explicit coin operator. Finally, in Sec. IV we conclude with some remarks.

\section{TWO-DIMENSIONAL QUANTUM WALK}

In this section we show the equivalence between the alternate and Pauli walks for any arbitrary choice of coin operator in $\mathrm{SU}(2)$. We also propose modified Pauli walk and discuss its implementation in optical setting using $J$ plates and PBS for a particular choice of $\mathrm{SU}(2)$ parameters. To quantify hyperentanglement, we use entanglement negativity, which measures entanglement between any two of the three DoF.

Mathematical framework. Quantum walk in 2D can be implemented using a 2D coin operator and shift operators in $x$ and $y$-directions [16-18]. We define coin operator as $\hat{C}_{\sigma}$ [Eq. (1)], and shift operators can be defined as

$$
\begin{aligned}
\hat{S}_{x}= & \sum_{x=-\infty}^{\infty}\left[|H\rangle\langle H|\otimes| x-1\rangle\langle x| \otimes \mathbb{1}_{y}\right. \\
& \left.+|V\rangle\langle V|\otimes| x+1\rangle\langle x| \otimes \mathbb{1}_{y}\right], \\
\hat{S}_{y}= & \sum_{y=-\infty}^{\infty}\left[|H\rangle\left\langle H\left|\otimes \mathbb{1}_{x} \otimes\right| y-1\right\rangle\langle y|\right. \\
& \left.+|V\rangle\left\langle V\left|\otimes \mathbb{1}_{x} \otimes\right| y+1\right\rangle\langle y|\right],
\end{aligned}
$$

where $\mathbb{1}_{x}$ and $\mathbb{1}_{y}$ are identity operators in $x$ and $y$ spaces, respectively. If $\left|\Psi_{\text {in }}\right\rangle$ represents the initial state, the evolution operator $\hat{O}$ corresponding to alternate walk is [18]

$$
\left|\Psi_{1}\right\rangle=\hat{S}_{x}\left[\hat{C}_{\sigma} \otimes \mathbb{1}_{x y}\right] \hat{S}_{y}\left[C_{\sigma}^{\dagger} \otimes \mathbb{1}_{x y}\right]\left|\Psi_{\text {in }}\right\rangle=\hat{O}\left|\Psi_{\text {in }}\right\rangle,
$$

where $\mathbb{1}_{x y}=\mathbb{1}_{x} \otimes \mathbb{1}_{y}$. This alternate walk evolution operator can also be implemented using just two shift operators, $\hat{S}_{x}$ and $\hat{S}_{\sigma}$, where

$$
\begin{aligned}
\hat{S}_{\sigma}= & {\left[\hat{C}_{\sigma} \otimes \mathbb{1}_{x y}\right] \hat{S}_{y}\left[\hat{C}_{\sigma}^{\dagger} \otimes \mathbb{1}_{x y}\right] } \\
= & \sum_{y}\left[\left|u_{1}\right\rangle\left\langle u_{1}\left|\otimes \mathbb{1}_{x} \otimes\right| y-1\right\rangle\langle y|\right. \\
& \left.+\left|u_{2}\right\rangle\left\langle u_{2}\left|\otimes \mathbb{1}_{x} \otimes\right| y+1\right\rangle\langle y|\right] .
\end{aligned}
$$

The states $\left|u_{1}\right\rangle$ and $\left|u_{2}\right\rangle$ denote the first and second column vectors of $\hat{C}_{\sigma}$ [see Eq. (1)], respectively. In any physical system with provision to directly realize $\hat{S}_{x}$ and $\hat{S}_{\sigma}$, without explicit use of coin operation, we can realize a 2D DTQW. When $\left\{\left|u_{1}\right\rangle,\left|u_{2}\right\rangle\right\}$ are the eigenvectors of the Pauli matrices,

$$
\sigma_{1}=\left[\begin{array}{ll}
0 & 1 \\
1 & 0
\end{array}\right] \quad \text { or } \quad \sigma_{2}=\left[\begin{array}{cc}
0 & -i \\
i & 0
\end{array}\right]
$$


the evolution operator $\hat{S}_{x} \hat{S}_{\sigma}$ readily implements the Pauli walk [18]. Therefore, the operator $\hat{S}_{x} \hat{S}_{\sigma}$ can be thought of as a generalized Pauli walk and its evolution can be given by

$$
\left|\Psi_{1}\right\rangle=\hat{S}_{x} \hat{S}_{\sigma}\left|\Psi_{\text {in }}\right\rangle=\hat{O}\left|\Psi_{\text {in }}\right\rangle .
$$

Shift operators $\hat{S}_{x}$ and $\hat{S}_{y}$ given in Eqs. (4) and (5) shift the position vector without changing the coin state vectors. However, we can also define a modified shift operator which induces a flip in the coin state vector along with the shift in position vector. For example, we can define

$$
\begin{aligned}
\hat{S}_{y}^{\prime}= & {\left[\sigma_{1} \otimes \mathbb{1}_{x y}\right] \hat{S}_{y}=\sum_{y=-\infty}^{\infty}\left[|V\rangle\left\langle H\left|\otimes \mathbb{1}_{x} \otimes\right| y-1\right\rangle\langle y|\right.} \\
& \left.+|H\rangle\left\langle V\left|\otimes \mathbb{1}_{x} \otimes\right| y+1\right\rangle\langle y|\right],
\end{aligned}
$$

and $\hat{S}_{x}^{\prime}$ defined likewise. Because of the bit-flip symmetry [37], it can be shown that both $\hat{O}$ and

$$
\hat{O}^{\prime} \equiv \hat{S}_{x}\left[\hat{C}_{\sigma} \otimes \mathbb{1}_{x y}\right] \hat{S}_{y}^{\prime}\left[C_{\sigma}^{\dagger} \otimes \mathbb{1}_{x y}\right]\left|\Psi_{\text {in }}\right\rangle
$$

lead to the equivalent evolution of the initial state $\left|\Psi_{\text {in }}\right\rangle$ in Eq. (6). By equivalent evolution, we mean that both $\hat{O}$ and $\hat{O}^{\prime}$ lead to the same position probability distribution. Thus, we can also define modified versions of both alternate and generalized Pauli walks as

$$
\begin{gathered}
\left|\Psi_{1}\right\rangle=\hat{S}_{x}\left[\hat{C}_{\sigma} \otimes \mathbb{1}_{x y}\right] \hat{S}_{y}^{\prime}\left[\hat{C}_{\sigma}^{\dagger} \otimes \mathbb{1}_{x y}\right]\left|\Psi_{\text {in }}\right\rangle, \\
\text { and }\left|\Psi_{1}\right\rangle=\hat{S}_{x} \hat{S}_{\sigma}^{\prime}\left|\Psi_{\text {in }}\right\rangle,
\end{gathered}
$$

respectively, with

$$
\begin{aligned}
\hat{S}_{\sigma}^{\prime}= & {\left[\hat{C}_{\sigma} \otimes \mathbb{1}_{x y}\right] \hat{S}_{y}^{\prime}\left[\hat{C}_{\sigma}^{\dagger} \otimes \mathbb{1}_{x y}\right] } \\
= & \sum_{y}\left[\left|u_{2}\right\rangle\left\langle u_{1}\left|\otimes \mathbb{1}_{x} \otimes\right| y-1\right\rangle\langle y|\right. \\
& \left.+\left|u_{1}\right\rangle\left\langle u_{2}\left|\otimes \mathbb{1}_{x} \otimes\right| y+1\right\rangle\langle y|\right] .
\end{aligned}
$$

If we begin with the initial state

$$
\left|\Psi_{\text {in }}\right\rangle=\left(\cos \alpha|H\rangle+e^{i \beta} \sin \alpha|V\rangle\right) \otimes|x=0\rangle \otimes|y=0\rangle,
$$

then, after $n$ steps, the state will be of the form

$$
\begin{aligned}
\left|\Psi_{n}\right\rangle & =\left(\hat{S}_{x} \hat{S}_{\sigma}^{\prime}\right)^{n}\left|\Psi_{\text {in }}\right\rangle \\
& =\sum_{x, y}\left[\left(a_{x, y}^{(n)}|H\rangle+b_{x, y}^{(n)}|V\rangle\right) \otimes|x\rangle \otimes|y\rangle\right],
\end{aligned}
$$

where $a_{x, y}^{(n)}$ and $b_{x, y}^{(n)}$ are normalized complex coefficients. The recurrence relations between $a_{x, y}^{(n)}$ and $b_{x, y}^{(n)}$ are

$$
\begin{aligned}
a_{x, y}^{(n)}= & a_{x+1, y+1}^{(n-1)}\left(e^{i \zeta} \sin \theta\right)\left(e^{-i \xi} \cos \theta\right) \\
& +a_{x+1, y-1}^{(n-1)}\left(e^{i \xi} \cos \theta\right)\left(e^{-i \zeta} \sin \theta\right) \\
& +b_{x-1, y+1}^{(n-1)}\left(e^{-i \xi} \cos \theta\right)\left(e^{-i \xi} \cos \theta\right) \\
& +b_{x-1, y-1}^{(n-1)}\left(-e^{-i \zeta} \sin \theta\right)\left(e^{-i \zeta} \sin \theta\right),
\end{aligned}
$$

and

$$
\begin{aligned}
b_{x, y}^{(n)}= & a_{x+1, y+1}^{(n-1)}\left(e^{i \zeta} \sin \theta\right)\left(-e^{i \zeta} \sin \theta\right) \\
& +a_{x+1, y-1}^{(n-1)}\left(e^{i \xi} \cos \theta\right)\left(e^{i \xi} \cos \theta\right)
\end{aligned}
$$

$$
\begin{aligned}
& +b_{x-1, y+1}^{(n-1)}\left(e^{-i \xi} \cos \theta\right)\left(-e^{i \zeta} \sin \theta\right) \\
& +b_{x-1, y-1}^{(n-1)}\left(-e^{-i \zeta} \sin \theta\right)\left(e^{i \xi} \cos \theta\right) .
\end{aligned}
$$

The above described state evolution after $n$ steps is in superposition of the tensor products of the three Hilbert spaces, namely, coin Hilbert space $\mathcal{H}_{c}$, and two position Hilbert spaces $\mathcal{H}_{p_{x}}$ and $\mathcal{H}_{p_{y}}$ associated with the dynamics. The interwinding coefficients of the state vectors after evolution clearly indicate that Eq. (16) is hyperentangled [1].

Optical realization. It is possible to realize the shift operators in Eq. (13) without an explicit coin operation using passive optical devices PBS and $J$ plates on polarization and OAM DoF, respectively. Operator $\hat{S}_{x}\left(\hat{S}_{x, \text { pos }}\right.$ from now on, with "pos" referring to the position DoF) can be readily realized using the PBS, which reflect horizontal polarization and transmit vertical polarization. Operator $\hat{S}_{\sigma}^{\prime}\left(\hat{S}_{\sigma, \text { OAM }}^{\prime}\right)$ can be realized using a $J$ plate.

To understand the action of $J$ plate let us consider the light field $\psi(r, \phi ; z)$ propagating in the $z$ direction, where $r=\sqrt{x^{\prime 2}+y^{\prime 2}}$ and $\phi=\tan ^{-1}\left(y^{\prime} / x^{\prime}\right)$ with $\left(x^{\prime}, y^{\prime}\right)$ being the coordinates in the transverse plane. The light field $\psi(r, \phi ; z)$ carrying an OAM of $m \hbar$ per photon [36] can be written as

$$
\psi(r, \phi ; z) \propto A(r ; z) \exp (i m \phi),
$$

where $A(r ; z)$ denotes the amplitude profile and $\exp (\operatorname{im} \phi)$ denotes the phase profile. If this light field in the polarization state $\left|u_{1}\right\rangle$ passes through a $J$ plate [11] represented by a Jones matrix

$$
J(\phi)=e^{-i \phi}\left|u_{2}\right\rangle\left\langle u_{1}\left|+e^{i \phi}\right| u_{1}\right\rangle\left\langle u_{2}\right|,
$$

then its polarization vector (or Jones vector) will change to $\left|u_{2}\right\rangle$ and its phase profile will transform as $\exp [i(m-1) \phi]$, where $\left|u_{2}\right\rangle$ is the Jones vector orthogonal to $\left|u_{1}\right\rangle$. Likewise, the phase profile of the light field $\psi(r, \phi ; z)$ in the polarization state $\left|u_{2}\right\rangle$ will be transformed to $\exp [i(m+1) \phi]$ by the action of the $J$ plate, while the polarization state is changed to $\left|u_{1}\right\rangle$. Therefore, we find that the OAM of the light field has been reduced by $\hbar$ per photon in the former case, whereas it has been increased by $\hbar$ per photon in the latter case.

Now let us consider a single photon carrying an OAM of $m \hbar$ per photon in some polarization state. The $J$ plate can decrease (increase) the OAM of the incoming photon with Jones vector $\left|u_{1}\right\rangle\left(\left|u_{2}\right\rangle\right)$ by $\hbar$ per photon while simultaneously transforming the Jones vector of the photon to $\left|u_{2}\right\rangle\left(\left|u_{1}\right\rangle\right)$. In other words, a $J$ plate changes the OAM of the incoming single photon conditioned over the polarization states $\left\{\left|u_{1}\right\rangle,\left|u_{2}\right\rangle\right\}$. Since $\left|u_{1}\right\rangle$ and $\left|u_{2}\right\rangle$ are themselves functions of $(\xi, \zeta, \theta)$ [Eqs. (1) and (7)], $J(\phi)$ in Eq. (20) can also be written as

$$
J(\phi) \equiv J(\phi, \xi, \zeta, \theta) .
$$

Thus, the shift operator realizing this transformation will be [cf. Eq. (14)],

$$
\begin{aligned}
\hat{S}_{\sigma, \mathrm{OAM}}^{\prime}= & \sum_{m}\left[\left|u_{2}\right\rangle\left\langle u_{1}\left|\otimes \mathbb{1}_{x, \operatorname{pos}} \otimes\right| m-1\right\rangle\langle m|\right. \\
& \left.+\left|u_{1}\right\rangle\left\langle u_{2}\left|\otimes \mathbb{1}_{x, \operatorname{pos}} \otimes\right| m+1\right\rangle\langle m|\right] .
\end{aligned}
$$


(a)

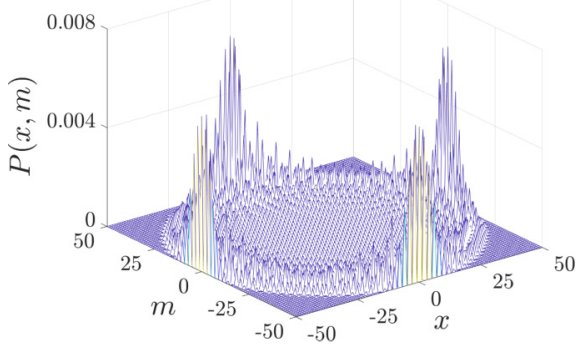

(b)

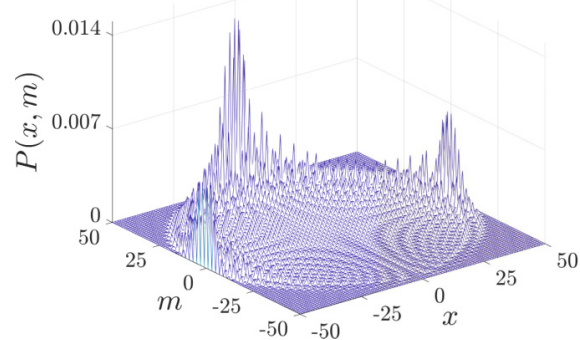

(c)

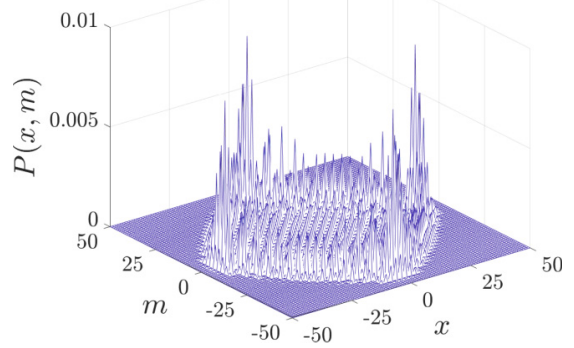

FIG. 1. Probability distribution $[P(x, m)]$ of the 2D DTQW without explicit coin operation in position and OAM DoF, beginning with an initial state $[(|H\rangle+|V\rangle) / \sqrt{2}] \otimes|x=0\rangle \otimes|y=0\rangle$, after 50 steps. (a) Modified Pauli walk using $q$ plates and PBS [see Eq. (23)]. Note that the probability distribution is identical for the Pauli walk [see Eqs. (9) and (24)] which is realized using $q$ - plates, PBS, and HWP. (b) Modified Pauli walk using $J$ plates and PBS for the choice $\left|u_{1}\right\rangle=[1,-1]^{T} / \sqrt{2}$ and $\left|u_{2}\right\rangle=[1,1]^{T} / \sqrt{2}$ in Eq. (23). (c) Modified Pauli walk using $J$ plates and PBS for the choice $\left|u_{1}\right\rangle=[1, \sqrt{3} i]^{T} / 2$ and $\left|u_{2}\right\rangle=[\sqrt{3},-i]^{T} / 2$ in Eq. (23).

For the special case when $\left|u_{1}\right\rangle=|R\rangle=[1,-i]^{T} / \sqrt{2}$ and $\left|u_{2}\right\rangle=|L\rangle=[1, i]^{T} / \sqrt{2}$, or equivalently for the choice $J(\phi, 0,-\pi / 2, \pi / 4), \hat{S}_{\sigma, \mathrm{OAM}}^{\prime}$ is realized using a $q$ plate [10]. With these, we find that a single photon in the initial state $\left|\Psi_{\text {in }}\right\rangle$, under the action of PBS and $J$ plates, will evolve as

$$
\begin{aligned}
\left|\Psi_{n}\right\rangle & =\left(\hat{S}_{x, \operatorname{pos}} \hat{S}_{\sigma, \mathrm{OAM}}^{\prime}\right)^{n}\left|\Psi_{\text {in }}\right\rangle \\
& =\sum_{x, m}\left[\left(a_{x, m}^{(n)}|H\rangle+b_{x, m}^{(n)}|V\rangle\right) \otimes|x\rangle \otimes|m\rangle\right],
\end{aligned}
$$

where the normalized complex coefficients are iteratively related as in Eqs. (17) and (18) with the position label $y$ being replaced by the OAM label $m$.

We can also realize

$$
\begin{aligned}
\hat{S}_{\sigma, \mathrm{OAM}}= & \sum_{m}\left[\left|u_{1}\right\rangle\left\langle u_{1}\left|\otimes \mathbb{1}_{x, \text { pos }} \otimes\right| m-1\right\rangle\langle m|\right. \\
& \left.+\left|u_{2}\right\rangle\left\langle u_{2}\left|\otimes \mathbb{1}_{x, \text { pos }} \otimes\right| m+1\right\rangle\langle m|\right]
\end{aligned}
$$

[see Eq. (7)] using a $J$ plate with Jones matrix

$$
\begin{aligned}
\tilde{J}(\phi) & =e^{-i \phi}\left|u_{1}\right\rangle\left\langle u_{1}\left|+e^{i \phi}\right| u_{2}\right\rangle\left\langle u_{2}\right| \\
& \equiv \tilde{J}(\phi, \xi, \zeta, \theta) .
\end{aligned}
$$

However, in order to realize $\hat{S}_{\sigma, \mathrm{OAM}}$ with $\left|u_{1}\right\rangle=|R\rangle$ and $\left|u_{2}\right\rangle=|L\rangle$ using a $q$ plate instead of a $J$ plate, we will require an additional half waveplate (HWP).

The probability distribution of both Pauli and modified Pauli walks for $n=50$ steps beginning with an initial state $\left|\Psi_{\text {in }}\right\rangle=[(|H\rangle+|V\rangle) / \sqrt{2}] \otimes|x=0\rangle \otimes|m=0\rangle$ [that is, by substituting $\alpha=\pi / 4$ and $\beta=0$ in Eq. (15)] has been shown in Fig. 1. In Fig. 1(a) we have considered a modified Pauli walk using $q$ plates and PBS [see Eq. (23)]. Owing to bit-flip symmetry [37], the probability distribution for the Pauli walk - realized using $q$ plates, PBS, and HWP-will also be identical to that of Fig. 1(a) [Eqs. (9) and (24)]. In Figs. 1(b) and 1(c) we have considered a modified Pauli walk-realized using $J$ plates and PBS. The orthogonal state vectors $\left\{\left|u_{1}\right\rangle,\left|u_{2}\right\rangle\right\}$ for Figs. 1(b) and 1(c) were chosen to be

$$
\begin{aligned}
& \left\{[1,-1]^{T} / \sqrt{2},[1,1]^{T} / \sqrt{2}\right\}, \text { and } \\
& \left\{[1, \sqrt{3} i]^{T} / 2,[\sqrt{3},-i]^{T} / 2\right\},
\end{aligned}
$$

respectively.

\section{GENERATION OF HYPERENTANGLEMENT}

In this section we present an outline of the optical setup which can hyperentangle the incoming single photon in polarization, path, and OAM DoF and realize 2D DTQW. The hyperentanglement between the three DoF involved in the dynamics is quantified using entanglement negativity between the combination of the Hilbert spaces.

In Fig. 2 we present the schematic representation of the setup for optical implementation of both Pauli and modified Pauli walks. In the case of the modified Pauli walk, the shift operator $\hat{S}_{x, \text { pos }}$ is realized using a PBS, and $\hat{S}_{\sigma, \mathrm{OAM}}^{\prime}$ is realized using a $J$ plate for any given orthogonal set of vectors $\left\{\left|u_{1}\right\rangle,\left|u_{2}\right\rangle\right\}$ [see Eq. (23)]. To realize the Pauli walk, we just

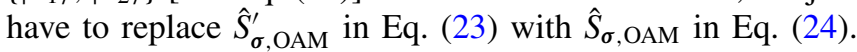
Clearly, $\hat{S}_{\sigma, \mathrm{OAM}}$ is also realized using a $J$ plate with the Jones matrix given in Eq. (25). When $\left\{\left|u_{1}\right\rangle,\left|u_{2}\right\rangle\right\}=\{|R\rangle,|L\rangle\}$, every $J$ plate can be replaced with a $q$ plate in the modified Pauli walk. On the other hand, every $J$ plate has to be replaced with a $q$ plate and a HWP to realize the Pauli walk.

While $\hat{S}_{x, \text { pos }}$ controls the path DoF of the single photon, $\hat{S}_{\sigma, \mathrm{OAM}}^{\prime}\left(\right.$ or $\hat{S}_{\sigma, \mathrm{OAM}}$ ) controls both polarization and OAM DoF of the same. Therefore, we do not need to explicitly use a coin operation to control the polarization DoF. This setup requires $n(n+1) / 2 \mathrm{PBS}$ and $n(n-1)+1 J$ plates to realize the modified Pauli walk (or Pauli walk) for $n$ steps. Here we have two remarks to make. First, the number of PBS and $J$ plates required to implement this type of quantum walk will scale quadratically with the number of steps. Second, when $\left\{\left|u_{1}\right\rangle,\left|u_{2}\right\rangle\right\}=\{|H\rangle,|V\rangle\}$, the evolved state is localized at the center and no spread is therefore observed.

The single photon, after $n$ steps, would have evolved in superposition of position and OAM space. Upon measurement it will collapse at any one of the detector units $D_{0}, D_{1}, D_{-1}, \ldots$, placed as shown in Fig. 2. Each detector unit contains a spatial light modulator, a single mode fiber, and a single photon detector (SPD). The measurement of the OAM DoF requires all three of these components [27,32], whereas the measurement of the path DoF requires just a SPD [34]. To realize the 2D DTQW, we note that the Hilbert space corresponding to the photon's path represents one spatial dimension, $x$ axis. Since the photon at each position $x$ can end up with an OAM value 


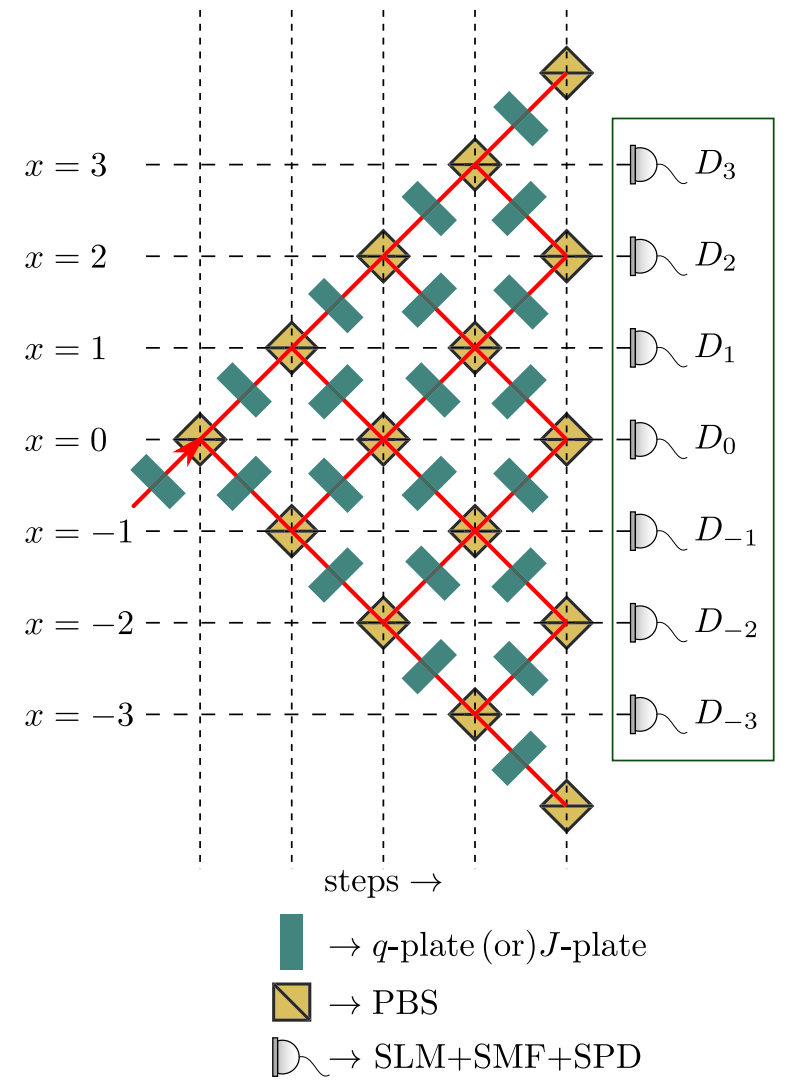

FIG. 2. Optical implementation of Pauli and modified Pauli walks. Both Pauli and modified Pauli walks with any orthogonal set of vectors $\left\{\left|u_{1}\right\rangle,\left|u_{2}\right\rangle\right\}$ can be realized using $J$ plates and PBS [see Eqs. (9), (23), and (24)]. When $\left\{\left|u_{1}\right\rangle,\left|u_{2}\right\rangle\right\}=\{|R\rangle,|L\rangle\}$, every $J$ plate can be replaced with a $q$ plate $(q$ plate and HWP) to realize the modified Pauli walk (Pauli walk). $D_{i}$ denotes a detector unit placed at the position $x=i$. Each detector unit consists of a spatial light modulator (SLM), a single mode fiber (SMF), and a single photon detector (SPD). A single photon is sent through a $q$ plate (or $J$ plate) to the PBS placed at $x=0$ (shown with an arrow). Here, $J$ plate implements either one of the shift operators $\hat{S}_{\sigma, \text { OAM }}^{\prime}$ [Eq. (22)] or $\hat{S}_{\sigma, \text { OAM }}$ [Eq. (24)], and PBS implements the shift operator $\hat{S}_{x \text {,pos }}$ [see Eq. (23)]. Evidently, no explicit 2D coin operation is necessary to implement these type of quantum walks.

of $y \hbar$ per photon, it represents the second spatial dimension, $y$ axis.

After $n$ steps, the single photon will be entangled in polarization, path, and OAM DoF. To quantify the amount of entanglement between any two DoF, we adopt a measure known as the entanglement negativity [38]. Here, we first partial trace out the density matrix $\left|\Psi_{n}\right\rangle\left\langle\Psi_{n}\right|$ with respect to the third DoF. After partial transpose of the resulting reduced density matrix we compute

$$
\mathcal{N}=\sum_{i} \frac{\left(\left|\lambda_{i}\right|-\lambda_{i}\right)}{2}
$$

where $\lambda_{i}$ 's are the eigenvalues of the partial transposed reduced density matrix. Evidently, $\mathcal{N}=0$ implies the reduced system is unentangled.
Now we present our numerically simulated results of a single photon passing through the optical setup schematically outlined in Fig. 2. The probability distribution of the evolved state after 50 steps for three different $J$-plate parameters $(\xi, \zeta, \theta)$ [see (21)] has been shown in Fig. 1. Note that $\hat{S}_{\sigma, \mathrm{OAM}}^{\prime}$ [Eq. (22)] with any $(\xi, \zeta, \theta)$ (or equivalently, $\left\{\left|u_{1}\right\rangle,\left|u_{2}\right\rangle\right\}$ ) can be realized using a $J$ plate. Nevertheless, $\hat{S}_{\sigma, \text { OAM }}^{\prime}$ with the choice $(\xi, \zeta, \theta)=(0,-\pi / 2, \pi / 4)$ can be implemented using a $q$ plate. In order to demonstrate that the three DoF are entangled, we first trace out the polarization DoF from the density matrix corresponding to $\left|\Psi_{n}\right\rangle$ [see Eq. (16)] and compute the entanglement negativity $\mathcal{N}$ [see Eq. (26)] corresponding to the partial transposed reduced density matrix. We then plot $\mathcal{N}$ against the increasing number of steps $n$ in Fig. 3 .

In frame (a) of Fig. 3 we have shown $\mathcal{N}$ between the path and OAM DoF as a function of the number of steps. As we increase the number of steps, $\mathcal{N}$ also increases linearly. The value $\mathcal{N}$ can be controlled using the $J$-plate parameters $(\xi, \zeta, \theta)$. If we partial trace any one of the spatial DoF (path or OAM DoF), $\mathcal{N}$ corresponding to the reduced density matrix between the polarization and OAM (or polarization and path) DoF reaches a steady value as we increase the number of steps [see frame (b) of Fig. 3] for various choices of the $J$-plate parameters $(\xi, \zeta, \theta)$. For instance, for the choice $(\xi, \zeta, \theta)=(0,-\pi / 2, \pi / 4)$, i.e., a $q$ plate, $\mathcal{N}$ between polarization and OAM (or path) DoF reaches a steady-state value 0.17927 after 25 steps, provided we begin with an initial state $[(|H\rangle+|V\rangle) / \sqrt{2}] \otimes|x=0\rangle \otimes|y=0\rangle$.

We now demonstrate how the $J$-plate parameters $(\xi, \zeta, \theta)$, beginning with an initial state, can be used to control the amount of entanglement between three DoF : polarization, path, and OAM. In other words, we demonstrate numerically how $\mathcal{N}$ can be controlled by tuning the $J$-plate parameters. In Fig. 4 we have shown how the negativity $\mathcal{N}$ between any two DoF varies with respect to the $J$-plate parameters $(\xi, \zeta, \theta)$. Here, we have allowed one of the three $J$-plate parameters $(\xi, \zeta, \theta)$ to vary while keeping the remaining two constant and plotted the respective $\mathcal{N}$ values. Furthermore, $\mathcal{N}$ between any two DoF has been computed after evolving the quantum walk for 25 steps. In frame (a) of Fig. $4 \mathcal{N}$ between path and OAM DoF has been plotted as a function of one of the three $J$-plate parameters. And in frame (b) of Fig. $4 \mathcal{N}$ between polarization and OAM (or path) DoF has been obtained as a function of the same. By keeping $\xi=\zeta=\pi / 2$ and varying $\theta$ in steps of $\pi / 180$ from 0 through $\pi / 2$, we obtain a black curve as shown in Fig. 4. Likewise, keeping $\zeta=-\pi / 2$ and $\theta=\pi / 4$ and varying $\xi$ in steps of $\pi / 180$ from 0 through $\pi / 2$, we obtain a red curve as shown in Fig. 4. The entanglement negativity $\mathcal{N}$ corresponding to the $q$ plate is encircled in both (a) and (b).

\section{CONCLUSION}

To summarize, we have proposed a passive optical setupusing $J$ plates or $q$ plates, and PBS - to hyperentangle an incoming single photon in polarization, path, and OAM DoF. We have shown that this optical setup can be efficiently used to simulate the 2D DTQW with variable evolution parameters (modified Pauli walk) without explicitly using a quantum coin operation. The evolved state has been numerically shown 

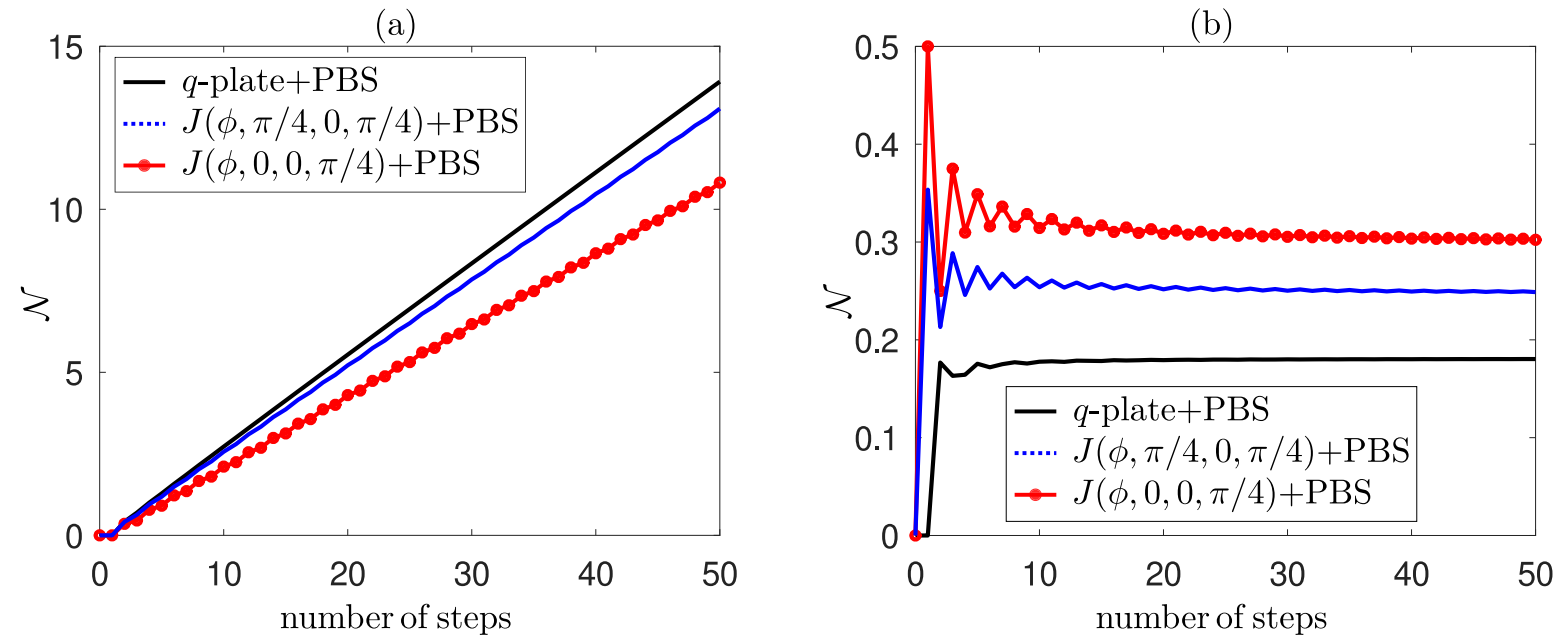

FIG. 3. Entanglement negativity $\mathcal{N}$ [see Eq. (26)] plotted against the increasing number of steps between two DoF for various $J$-plate parameters $(\xi, \zeta, \theta)$ [see (21)]. Here, $J(\phi, 0,-\pi / 2, \pi / 4)$ represents a $q$ plate, and the initial state is $[(|H\rangle+|V\rangle) / \sqrt{2}] \otimes|x=0\rangle \otimes|y=0\rangle$. (a) $\mathcal{N}$ between path and OAM DoF. (b) $\mathcal{N}$ between polarization and OAM DoF. Note that $\mathcal{N}$ between polarization and path DoF is identical to that of (b).

to be hyperentangled in polarization, path, and OAM DoF. The amount of entanglement between any two of the three DoF has been computed using entanglement negativity. It was observed that the entanglement negativity increased linearly between the path and OAM DoF, whereas the same between the polarization and path (or OAM) DoF remained constant after few number of steps due to the bound on the dimension of the coin space. The amount of entanglement between any two of the three DoF and the hyperentanglement in the system can be controlled by varying the $J$-plate parameters. The ability to control and engineer the dynamics of quantum walks using optical components can also play an important role in the realization of non-Markovian quantum channels [39] and the study of open quantum systems.

\section{ACKNOWLEDGMENTS}

P.A.A.Y. would like to thank Abhaya S. Hegde for useful discussions on hyperentangled states. P.A.A.Y. and C.M.C. acknowledge the support from the Office of Principal Scientific Advisor to Government of India, Project No. Prn.SA/QSim/2020 and Interdisciplinary Cyber Physical Systems (ICPS) program of the Department of Science and Technology, India, Grant No. DST/ICPS/QuST/Theme$1 / 2019 / 1$. (a)

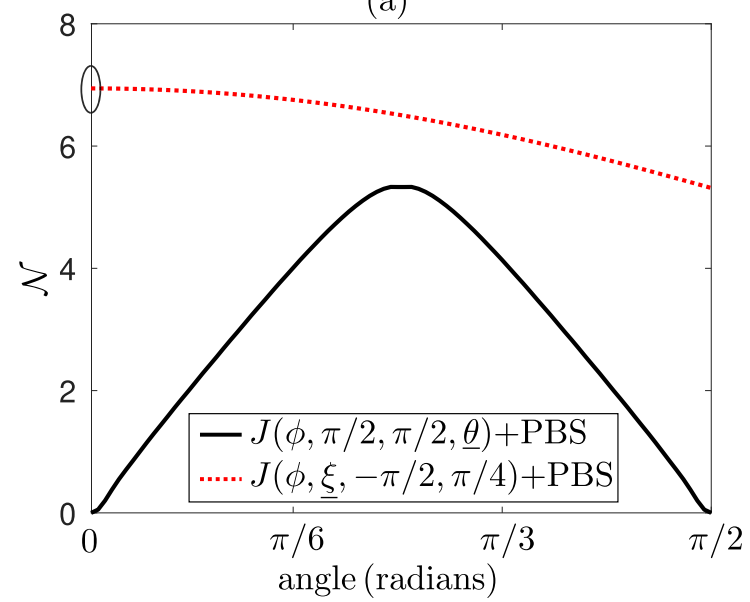

(b)

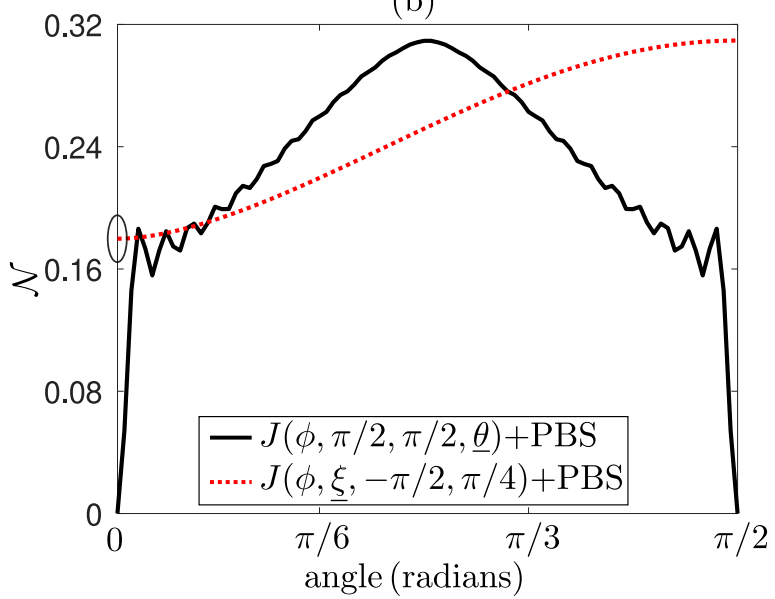

FIG. 4. Entanglement negativity $\mathcal{N}$ [see Eq. (26)] between any two of the three DoF plotted against any one of the three $J$-plate parameters $(\xi, \zeta, \theta)[$ see $(21)]$ after 25 steps. The initial state was taken to be $[(|H\rangle+|V\rangle) / \sqrt{2}] \otimes|x=0\rangle \otimes|y=0\rangle$. In (a), $\mathcal{N}$ between path and OAM DoF is considered, whereas in (b) that between polarization and OAM DoF is considered. In both (a) and (b) the black curve was obtained for the $J(\phi, \pi / 2, \pi / 2, \underline{\theta})$ and PBS combination, where $\underline{\theta}$ denotes that $\theta$ is varied from 0 through $\pi / 2$ in steps of $\pi / 180$. And the red curve was obtained for the $J(\phi, \xi,-\pi / 2, \pi / 4)$ and PBS combination, where $\xi$ denotes that $\xi$ is varied from 0 through $\pi / 2$ in steps of $\pi / 180$. Note that the choice $J(\phi, 0,-\pi / 2, \pi / 4)$ represents a $q$ plate and is encircled in both (a) and (b). 
[1] P. G. Kwiat, J. Mod. Opt. 44, 2173 (1997).

[2] J. T. Barreiro, N. K. Langford, N. A. Peters, and P. G. Kwiat, Phys. Rev. Lett. 95, 260501 (2005).

[3] T.-M. Zhao, Y. S. Ihn, and Y.-H. Kim, Phys. Rev. Lett. 122, 123607 (2019).

[4] J. T. Barreiro, T.-C. Wei, and P. G. Kwiat, Nat. Phys. 4, 282 (2008).

[5] Y.-B. Sheng and F.-G. Deng, Phys. Rev. A 81, 032307 (2010).

[6] Y.-B. Sheng, F.-G. Deng, and G. L. Long, Phys. Rev. A 82, 032318 (2010).

[7] Y.-B. Sheng and F.-G. Deng, Phys. Rev. A 82, 044305 (2010).

[8] X. M. Hu, C. X. Huang, Y.-B. Sheng, L. Zhou, B.-H. Liu, Y. Guo, C. Zhang, W.-B. Xing, Y.-F. Huang, C.-F. Li, and G.-C. Guo, Phys. Rev. Lett. 126, 010503 (2021).

[9] A. T. O’Neil, I. MacVicar, L. Allen, and M. J. Padgett, Phys. Rev. Lett. 88, 053601 (2002).

[10] L. Marrucci, C. Manzo, and D. Paparo, Phys. Rev. Lett. 96, 163905 (2006).

[11] R. C. Devlin, A. Ambrosio, N. A. Rubin, J. B. Mueller, and F. Capasso, Science 358, 896 (2017).

[12] S. E. Venegas-Andraca, Quantum Inf. Process. 11, 1015 (2012).

[13] C. M. Chandrashekar, R. Srikanth, and R. Laflamme, Phys. Rev. A 77, 032326 (2008).

[14] R. Simon and N. Mukunda, Phys. Lett. A 143, 165 (1990).

[15] B. Tregenna, W. Flanagan, R. Maile, and V. Kendon, New J. Phys. 5, 83 (2003).

[16] C. Di Franco, M. Mc Gettrick, and T. Busch, Phys. Rev. Lett. 106, 080502 (2011).

[17] C. M. Chandrashekar, S. Banerjee, and R. Srikanth, Phys. Rev. A 81, 062340 (2010).

[18] C. M. Chandrashekar and T. Busch, J. Phys. A: Math. Theor. 46, 105306 (2013).

[19] C. A. Ryan, M. Laforest, J.-C. Boileau, and R. Laflamme, Phys. Rev. A 72, 062317 (2005).

[20] M. Karski, L. Förster, J.-M. Choi, A. Steffen, W. Alt, D. Meschede, and A. Widera, Science 325, 174 (2009).

[21] A. Schreiber, K. N. Cassemiro, V. Potoček, A. Gábris, P. J. Mosley, E. Andersson, I. Jex, and C. Silberhorn, Phys. Rev. Lett. 104, 050502 (2010).
[22] A. Crespi, R. Osellame, R. Ramponi, V. Giovannetti, R. Fazio, L. Sansoni, F. De Nicola, F. Sciarrino, and P. Mataloni, Nat. Photonics 7, 322 (2013).

[23] M. A. Broome, A. Fedrizzi, B. P. Lanyon, I. Kassal, A. AspuruGuzik, and A. G. White, Phys. Rev. Lett. 104, 153602 (2010).

[24] P. Zhang, X.-F. Ren, X.-B. Zou, B.-H. Liu, Y.-F. Huang, and G.-C. Guo, Phys. Rev. A 75, 052310 (2007).

[25] H. Schmitz, R. Matjeschk, C. Schneider, J. Glueckert, M. Enderlein, T. Huber, and T. Schaetz, Phys. Rev. Lett. 103, 090504 (2009).

[26] F. Zähringer, G. Kirchmair, R. Gerritsma, E. Solano, R. Blatt, and C. F. Roos, Phys. Rev. Lett. 104, 100503 (2010).

[27] F. Cardano, F. Massa, H. Qassim, E. Karimi, S. Slussarenko, D. Paparo, C. de Lisio, F. Sciarrino, E. Santamato, R. W. Boyd et al., Sci. Adv. 1, e1500087 (2015).

[28] S. K. Goyal, F. S. Roux, A. Forbes, and T. Konrad, Phys. Rev. Lett. 110, 263602 (2013).

[29] B. Sephton, A. Dudley, G. Ruffato, F. Romanato, L. Marrucci, M. Padgett, S. Goyal, F. Roux, T. Konrad, and A. Forbes, PLoS ONE 14, e0214891 (2019).

[30] H. Tang, X.-F. Lin, Z. Feng, J.-Y. Chen, J. Gao, K. Sun, C.-Y. Wang, P.-C. Lai, X.-Y. Xu, Y. Wang et al., Sci. Adv. 4, eaat3174 (2018).

[31] A. D’Errico, F. Cardano, M. Maffei, A. Dauphin, R. Barboza, C. Esposito, B. Piccirillo, M. Lewenstein, P. Massignan, and L. Marrucci, Optica 7, 108 (2020).

[32] P. Zhang, B.-H. Liu, R.-F. Liu, H.-R. Li, F.-L. Li, and G.-C. Guo, Phys. Rev. A 81, 052322 (2010).

[33] H. Jeong, M. Paternostro, and M. S. Kim, Phys. Rev. A 69, 012310 (2004).

[34] B. Do, M. L. Stohler, S. Balasubramanian, D. S. Elliott, C. Eash, E. Fischbach, M. A. Fischbach, A. Mills, and B. Zwickl, J. Opt. Soc. Am. B 22, 499 (2005).

[35] W.-C. Gao, C. Cao, X.-F. Liu, T.-J. Wang, and C. Wang, OSA Continuum 2, 1667 (2019).

[36] L. Allen, M. W. Beijersbergen, R. J. C. Spreeuw, and J. P. Woerdman, Phys. Rev. A 45, 8185 (1992).

[37] C. M. Chandrashekar, R. Srikanth, and S. Banerjee, Phys. Rev. A 76, 022316 (2007).

[38] G. Vidal and R. F. Werner, Phys. Rev. A 65, 032314 (2002).

[39] J. Naikoo, S. Banerjee, and C. M. Chandrashekar, Phys. Rev. A 102, 062209 (2020). 